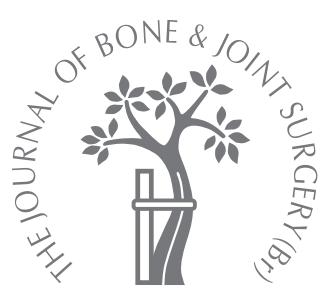

D. J. Beard,

H. Pandit,

S. Ostlere,

C. Jenkins,

C. A. F. Dodd,

D. W. Murray

From the Nuffield

Orthopaedic Centre

NHS Trust, Oxford,

England

D. J. Beard, DPhil, University

Research Lecturer and Fellow

In. Pandit, FRCS(Orth),

Clinical Research Fellow

a S. Ostlere, FRCR, Consultant

Radiologist

C. Jenkins, MSc, Senior

Research Physiotherapist

C. A. F. Dodd, FRCS,

Consultant Orthopaedic

Surgeon

D. W. Murray, FRCS(Orth),

Professor of Orthopaedics

Department of Orthopaedic

Surgery

University of Oxford, Nuffield

Department of Orthopaedic

Surgery, Nuffield Orthopaedic

Centre, Headington, Oxford,

OX3 7LD, UK.

Correspondence should be sent to Dr D. J. Beard; e-mail:

david.beard@ndos.ox.ac.uk

(C)2007 British Editorial Society of Bone and Joint Surgery

doi:10.1302/0301-620X.89B12.

$19260 \$ 2.00$

$J$ Bone Joint Surg $[\mathrm{Br}]$ 2007;89-B:1602-7.

Received 1 February 2007;

Accepted after revision 7

August 2007

\title{
Pre-operative clinical and radiological assessment of the patellofemoral joint in unicompartmental knee replacement and its influence on outcome
}

Anterior knee pain and/or radiological evidence of degeneration of the patellofemoral joint are considered to be contraindications to unicompartmental knee replacement. The aim of this study was to determine whether this is the case.

Between January 2000 and September 2003, in 100 knees (91 patients) in which Oxford unicompartmental knee replacements were undertaken for anteromedial osteoarthritis, pre-operative anterior knee pain and the radiological status of the patellofemoral joint were defined using the Altman and Ahlback systems. Outcome was evaluated at two years with the Oxford knee score and the American Knee Society score.

Pre-operatively 54 knees (54\%) had anterior knee pain. The clinical outcome was independent of the presence or absence of pre-operative anterior knee pain. Degenerative changes of the patellofemoral joint were seen in 54 patients $(54 \%)$ on the skyline radiographs, including ten knees (10\%) with joint space obliteration. Patients with medial patellofemoral degeneration had a similar outcome to those without. For some outcome measures patients with lateral patellofemoral degeneration had a worse score than those without, but these patients still had a good outcome, with a mean Oxford knee score of 37.6 (SD 9.5). These results show that neither anterior knee pain nor radiologically-demonstrated medial patellofemoral joint degeneration should be considered a contraindication to Oxford unicompartmental knee replacement. With lateral patellofemoral degeneration the situation is less well defined and caution should be observed.

The indications for unicompartmental knee replacement (UKR) are not universally agreed. Kozinn and Scott, in $1988,{ }^{1}$ stated that anterior knee pain and degenerative changes of the patellofemoral joint were contraindications, and despite the lack of supporting evidence many surgeons follow this intuitive recommendation. The practice of excluding these patients means that few data are available to challenge this stance.

The patellofemoral joint can be assessed both pre- and intra-operatively, but we are not aware of any study that has determined whether preoperative assessment is of value in determining patient suitability for UKR. It is generally considered that the presence of anterior knee pain and evidence of arthritis of the patellofemoral joint on skyline radiographs is important in this assessment.

The relationship between intra-operative assessment of the patellofemoral joint and outcome was assessed in a study of 125 bicompartmental Oxford knees by Goodfellow and O'Connor ${ }^{2}$ who found no relationship between the state of the patellofemoral joint as seen at operation and the outcome. Because their study involved a bicompartmental procedure we have recently undertaken a much larger study of UKR which came to the same conclusion. ${ }^{3}$ On the basis of the original study, Goodfellow et $\mathrm{al}^{4}$ recommended that the state of the patellofemoral joint should be ignored when deciding whether or not to implant a UKR, and we have adhered to these recommendations.

As this practice remains controversial, it is necessary to investigate further the value of preoperative assessment of the patellofemoral joint in UKR. For this reason we now take skyline radiographs of the patellofemoral joint and the patients are questioned by an independent observer about the site of their pain. The aim of this study was to establish whether the clinical practice of ignoring anterior knee pain and/or radiological evidence of degeneration of the patellofemoral joint is correct.

\section{Patients and Methods}

This study includes 91 patients (100 knees) who underwent a medial Oxford UKR (Biomet UK, Swindon, United Kingdom) inserted 
between January 2000 and September 2003. This cohort represents a subset of our main UKR study group reported separately ${ }^{3}$ and includes those patients who underwent more detailed questioning about their pain and also had skyline radiographs taken. A complete set of data was available two years post-operatively. The mean age of the patients at the time of surgery was 69 years (53 to 89). There were 46 women and 45 men, who underwent 82 unilateral and 9 staged bilateral procedures. All patients had anteromedial osteoarthritis (OA) and did not contravene the recommended contra-indications for the procedure which include failed upper tibial osteotomy, insufficiency of the collateral or anterior cruciate ligaments, a fixed varus deformity (not passively correctable) of greater than $15^{\circ}$ and a flexion deformity of greater than $15^{\circ}$. The presence of anterior knee pain and radiological evidence of degenerative change at the patellofemoral joint were not considered to be contraindications. The only exception occurred if a patient was found intra-operatively to have severe lateral OA of the patellofemoral joint with bone loss, grooving and eburnation, in which case a total knee replacement (TKR) was performed. The standard minimally-invasive surgical technique was used, with an incision from the medial pole of the patella to the tibial tuberosity, and without patellar dislocation. ${ }^{5,6}$

Data were collected prospectively with all patients assessed pre-operatively using the American Knee Society score $(\mathrm{AKSS})^{7}$ and Oxford knee score (OKS). ${ }^{8}$ The OKS was scored from 0 to 48 , where 48 is the best possible outcome. Patients were also asked to specify whether their knee pain was medial, anterior, lateral, or generalised. The radiological assessment included a skyline view taken with the knee flexed at approximately $30^{\circ}$. Both medial and lateral aspects of the patellofemoral joint were assessed to determine the presence of degenerative changes.

The radiological assessment was performed by an independent radiologist $(\mathrm{SO})$. The radiographs were scored for severity of arthritis using the Ahlback ${ }^{9}$ grading and Altman ${ }^{10}$ scoring system. The Ahlback ${ }^{9}$ grade measures the severity of cartilage and bone loss: 0 , normal, I, joint space narrowing, II, joint space obliteration, III, bone destruction $<5 \mathrm{~mm}$, IV, bone destruction $>5 \mathrm{~mm}$. We also assessed whether the joint was subluxed or not (grade V). The Altman score ${ }^{10}$ assesses a variety of arthritic characteristics, including osteophytosis, joint space narrowing, subchondral sclerosis and bone destruction. Each variable is scored from 0 to 3 , with a higher score indicating increasing severity. Thus the best score possible is 0 , and the worst is 12 .

Patients were assessed clinically two years after UKR by an independent observer $(\mathrm{CJ})$ who was blinded to the radiological findings and the pre- and intra-operative findings. They were asked whether they had pain, and if so, where it was sited, and scoring with the AKSS and OKS was repeated. Particular note was made of question 12 from the OKS scored 0 to 4 , which evaluates the ability to walk downstairs and provides further detail regarding the patellofemoral joint.

Data on patients with primary medial compartment OA who had undergone cemented Oxford UKR were used to calculate the sample size. A three-point difference on the OKS is considered clinically relevant, and existing data suggested a standard deviation of seven points. Using Altman's nomogram,${ }^{11}$ the sample size was calculated to be 85 for a power of $90 \%$, with an $\alpha$ significance level of 0.05 .

In order to assess the effect of pre-operative anterior knee pain on the clinical outcome, patients were divided into two groups according to whether or not they had such pain. In order to examine the effect of severity of degeneration of the patellofemoral joint from the radiographs on clinical outcome, both the Ahlback ${ }^{9}$ grade and Altman ${ }^{10}$ score were evaluated independently. The Ahlback grade was subdivided according to whether knees had joint space obliteration, with an Ahlback grading II or more, or not, with an Ahlback grading 0 or I. For a more general characterisation of arthritis the Altman score was used. Patients were considered to have no or minor degenerative changes with Altman scores 0 and 1 , whereas those with Altman score 2 or more were classified as having degenerative change. Association between the raw Altman score (severity grade) and the outcome was also examined.

The two-year post-operative clinical scores as measured by the absolute OKS, were used as the primary outcome variable throughout, although analysis was also performed on the other outcome scores, including the relative change over time for each variable. Relative change in outcome, or improvement, takes account of the variability in preoperative functional status. Scores were presented as mean values with standard deviations (SD). Incidence data were reported using frequencies, and two-group Mann-Whitney tests were performed to test respective hypotheses where data were found to be non-parametric. Independent $t$-tests were performed on parametric normally distributed data. Both Spearman's and Pearson's correlation coefficients were calculated, depending on the level of data, and scatterplots were used to examine any associations. Statistical tests were carried out using SPSS software (SPSS Inc., Chicago, Illinois) with statistical significance set at $\mathrm{p}<0.05$.

\section{Results}

Anterior knee pain. Of 100 knees, $80 \quad(80 \%)$ had preoperative pain localised to the medial aspect of the knee, 54 $(54 \%)$ had anterior pain, three $(3 \%)$ had lateral pain, and nine $(9 \%)$ had generalised pain. The mean pre-operative OKS in the 54 knees with anterior pain was 22.7 (SD 7.5) and was similar to the mean OKS in the 46 patients (46\%) without pre-operative anterior knee pain at 24.4 (SD 7.3). Only one patient who had pre-operative anterior knee pain also had anterior knee pain at two years. This patient had minimal degeneration of the patellofemoral joint (Ahlback grade 0 , Altman score 1 for each patellofemoral joint location). This patient has subsequently been reviewed at three 
Table I. Influence of pre-existing anterior knee pain on clinical outcome at two years in 100 knees (OKS, Oxford knee score; AKSS, American Knee Society score; SD, standard deviation)

\begin{tabular}{lccc}
\hline & $\begin{array}{l}\text { Presence of pre-operative } \\
\text { anterior knee pain }(\mathbf{n}=\mathbf{5 4})\end{array}$ & $\begin{array}{l}\text { Absence of pre-operative } \\
\text { anterior knee pain }(\mathbf{n}=\mathbf{4 6})\end{array}$ & p-value* $^{*}$ \\
\hline Mean OKS (SD) & $40.9(6.6)$ & $39.8(7.7)$ & 0.49 \\
Mean change in OKS (SD) & $18.1(7.9)$ & $15.3(8.3)$ & 0.11 \\
Mean OKS question 12 (SD) & $3.6(0.6)$ & $3.6(0.6)$ & 0.61 \\
Mean AKSS (SD) & $90.7(13.1)$ & $91.0(9.6)$ & 0.28 \\
\hline
\end{tabular}

* Mann-Whitney U test

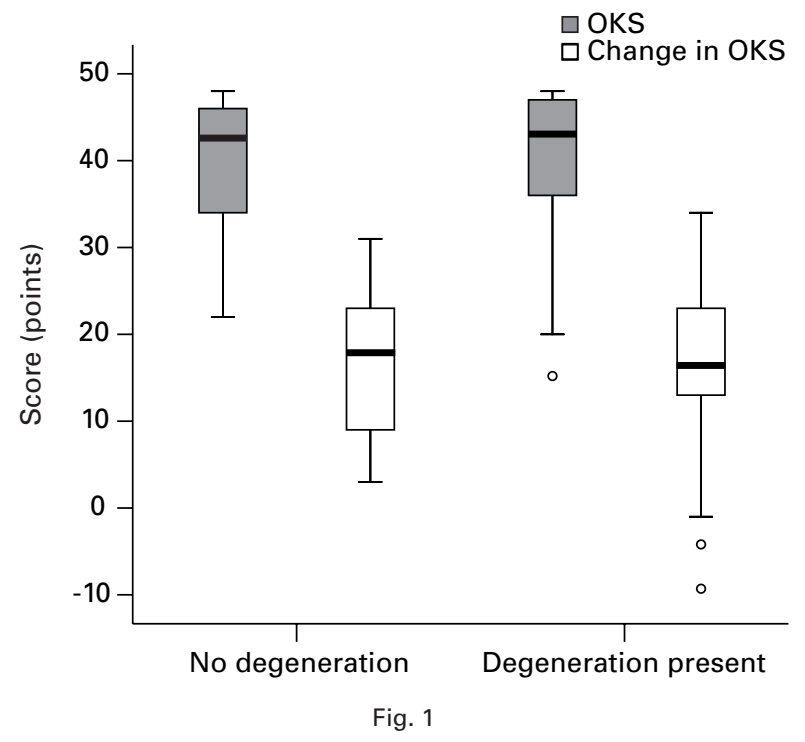

Outcome in terms of absolute Oxford knee score (OKS) and change in OKS at two years, with or without patellofemoral joint degeneration at any location within the joint, assessed radiologically using Altman scoring in 100 knees. The circles indicate outliers.

years and the pain has settled. No statistically significant difference was found between the groups with or without pre-operative anterior knee pain when the two-year absolute values of clinical outcome, the OKS, AKSS knee, and question 12 (Q12) of the OKS were compared (Table I). Similarly, there was no statistically significant difference in the improvement in function when relative change in outcome was examined between the two groups (Table I).

Grade of degeneration of the patellofemoral joint (Altman). Of 100 knees, $54(54 \%)$ had some evidence of arthritic degeneration of the patellofemoral joint (Altman 2 or more). In 45 knees (45\%), medial patellofemoral joint changes were noted, whereas $20(20 \%)$ had lateral changes and both medial and lateral changes were seen in patellofemoral joints in 11 knees $(11 \%)$.

Two types of analysis were performed to investigate the influence of degeneration of the patellofemoral joint outcome: whether the presence (Altman 2 or more) or absence of OA influenced the outcome, and the association between severity of degeneration (Altman score) and outcome.

There was no statistically significant difference in outcome (all variables) at two years between patients with evidence of degeneration anywhere in the patellofemoral joint and those with normal or nearly normal joints (Fig. 1) (Table II). Consideration was given to whether the patellofemoral joint degeneration was medial or lateral. Patients with medial degeneration of the patellofemoral joint did not have significantly different outcomes from those without patellofemoral joint degeneration for any outcome variable (Table II). In fact, outcome scores for knees with medial patellar degeneration were consistently, albeit not significantly, higher than those without degenerative changes. In contrast, patients with lateral patellar degeneration had statistically significantly lower values for absolute OKS, improvement in OKS and Q12 of the OKS than those with normal joints (Table II). Despite the lower values, the patients with lateral degeneration had good clinical outcome, with a mean OKS of 37.6 (SD 9.5) and mean AKS score of 90.4 (SD 10.9). They also had a substantial improvement in the mean OKS to 13.1 (SD 8.1)

There was no significant association between outcome determined by the absolute OKS or by the change in OKS and the severity of degeneration assessed by the Altman scoring of the skyline view of the medial patellar facet (Spearman's correlation coefficient, $r=0.11, p=0.3$ and $\mathrm{r}=0.1, \mathrm{p}=0.2$ respectively) (Fig. 2a). A similar lack of association was found for the lateral aspect of the patellofemoral joint. Although the plot revealed a slight trend for worse outcome with increased degeneration (Fig. 2b), the correlation was nominal (absolute OKS, $r=-0.05$ $(\mathrm{p}=0.6)$, change in OKS, $\mathrm{r}=-0.04(\mathrm{p}=0.6))$ and not significant.

Full-thickness cartilage loss (Ahlback). Of the 100 preoperative skyline views, six $(6 \%)$ had obliteration of the medial patellar joint space (Ahlback grade II or more) (Table III). In four knees (4\%) the lateral patellar joint space was obliterated. Only one patient had both medial and lateral joint space obliteration. Although the numbers are small, it was surprising to find that the six knees with obliteration of the medial joint space tended to have higher outcome scores at two years than the 94 knees with normal or nearly normal cartilage. This difference was statistically 
Table II. Influence of degenerative changes measured by the Altman score on clinical outcome at two years in 100 knees (OKS, Oxford knee score; AKSS, American Knee Society score; SD, standard deviation)

\begin{tabular}{|c|c|c|c|c|c|c|}
\hline \multirow[b]{2}{*}{ Outcome } & \multicolumn{3}{|l|}{ Medial } & \multicolumn{3}{|l|}{ Lateral } \\
\hline & Normal $(n=55)$ & Altman $\geq 2$ ( $n=45)$ & p-value ${ }^{*}$ & Normal $(n=80)$ & Altman $\geq 2(n=20)$ & p-value ${ }^{*}$ \\
\hline Mean OKS (SD) & $39.6(7.2)$ & $41.2(7.0)$ & 0.3 & $41.1(6.2)$ & $37.6(9.5)$ & 0.04 \\
\hline Mean OKS question 12 (SD) & $3.5(0.6)$ & $3.7(0.5)$ & 0.09 & $3.7(0.5)$ & $3.3(0.6)$ & 0.02 \\
\hline Mean AKSS knee (SD) & $89.3(13.4)$ & $92.8(8.6)$ & 0.4 & $90.1(11.8)$ & $90.4(10.9)$ & 0.8 \\
\hline Mean AKSS function (SD) & $85.3(15.5)$ & 89.9 (13.3) & 0.1 & $88.2(15.1)$ & $84.2(12.7)$ & 0.1 \\
\hline
\end{tabular}

${ }^{*}$ independent $t$-test

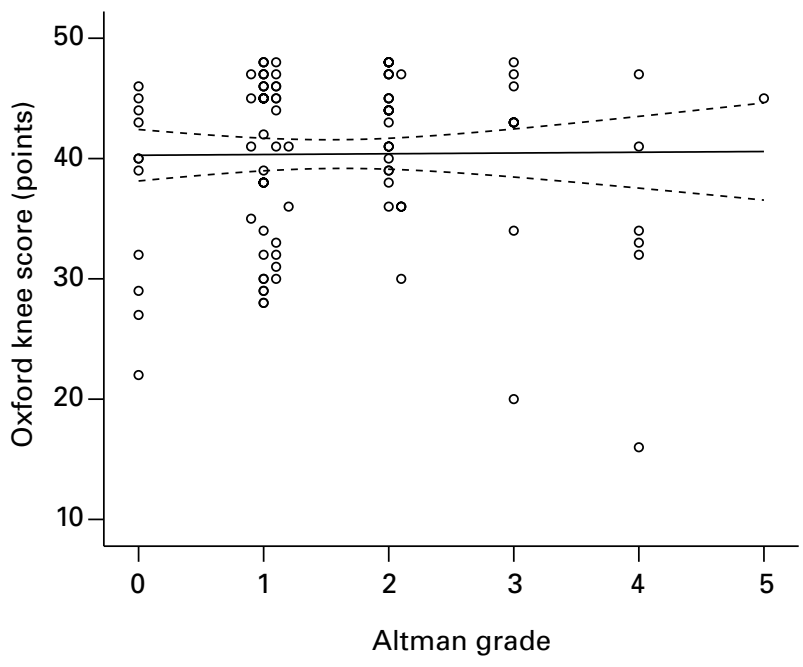

Fig. 2a

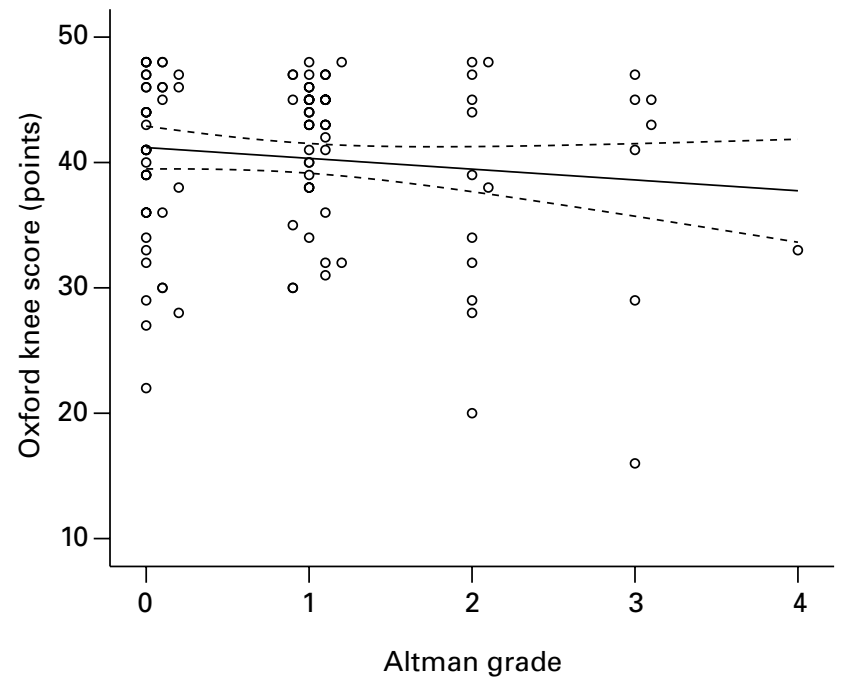

Fig. $2 b$

Relationship between outcome measured by absolute Oxford knee score at two years and severity of patellofemoral joint degeneration assessed from skyline radiographs using Altman scoring for a) the medial patellofemoral joint and b) the lateral patellofemoral joint in 100 knees undergoing medial unicondylar replacement. Linear regression trend (with 95\% confidence interval) is shown. The circles represent individual data points.

significant for the AKSS knee (independent $t$-test, $\mathrm{p}=0.01$ ) and Q12 of the OKS (independent $t$-test, $\mathrm{p}=0.001$ ). In contrast, and similarly to the Altman data, knees with obliteration of the lateral joint space tended to have poorer outcomes than those with normal lateral facets, but the effect was only significant for the relative change in OKS (independent $t$-test, $\mathrm{p}=0.03$ ). Again, despite the slight inferiority in outcome compared with knees without obliteration of the joint space, patients with lateral facet changes still achieved a good final outcome as measured by the OKS and change in OKS.

Relationship between anterior knee pain and damage of the patellofemoral joint. The relationship between anterior knee pain and grade of arthritis was examined using descriptive statistics. For each Altman score of degeneration occurring anywhere in the joint, approximately half the patients had pre-operative anterior knee pain and half did not (Fig. 3). A similar pattern existed for both the medial and the lateral patellofemoral joint when examined individually. Not surprisingly, no correlation was found between the presence of pre-operative anterior knee pain and the severity of radiologically-assessed degeneration anywhere in the joint (Spearman's correlation coefficient, $0.2(\mathrm{p}=0.55))$.

\section{Discussion}

This study has shown that neither pre-operative anterior knee pain nor radiological changes on the skyline patellar radiograph, with the exception of the lateral facet, compromise the outcome of UKR. There was anterior knee pain in approximately half the cases pre-operatively and, with one exception, the pain was alleviated after UKR. In the patient who had minor patellofemoral degenerative changes, the pain persisted for two years post-operatively and then resolved. In addition, no relationship existed between outcome and pre-operative anterior knee pain for any variable, from which we infer that when assessing the suitability of a patient for UKR anterior knee pain can be disregarded. 
Table III. Influence of joint space obliteration as measured by the Ahlback score on clinical outcome at two years in 100 knees (OKS, Oxford knee score; AKSS, American Knee Society score; SD, standard deviation)

\begin{tabular}{|c|c|c|c|c|c|c|}
\hline \multirow[b]{2}{*}{ Outcome } & \multicolumn{3}{|l|}{ Medial } & \multicolumn{3}{|l|}{ Lateral } \\
\hline & Normal $(n=94)$ & Ahlback $\geq$ II $(n=6)$ & p-value ${ }^{*}$ & Normal (n = 96) & Ahlback $\geq$ II $(n=4)$ & p-value ${ }^{*}$ \\
\hline Mean OKS (SD) & $40.2(7.1)$ & $42.3(6.9)$ & 0.5 & $40.5(7.15)$ & $38.0(8.2)$ & 0.5 \\
\hline Mean change in OKS (SD) & $16.8(8.1)$ & $18.3(9.5)$ & 0.7 & $17.1(8.2)$ & $11.0(3.6)$ & 0.03 \\
\hline Mean OKS question 12 (SD) & $3.6(0.6)$ & $4.0(0.0)$ & $0.001^{\dagger}$ & $3.7(0.5)$ & $3.5(0.6)$ & 0.6 \\
\hline Mean AKSS knee (SD) & $90.4(11.7)$ & $99.0(2.2)$ & $0.01^{\dagger}$ & $90.9(11.5)$ & $89.5(13.7)$ & 0.9 \\
\hline Mean AKSS function (SD) & $87.5(14.6)$ & $84.0(18.1)$ & 0.6 & $87.7(14.8)$ & $80.0(8.1)$ & 0.2 \\
\hline
\end{tabular}

* independent $t$-test

${ }^{\dagger}$ statistical significance showing a superior outcome in affected joints

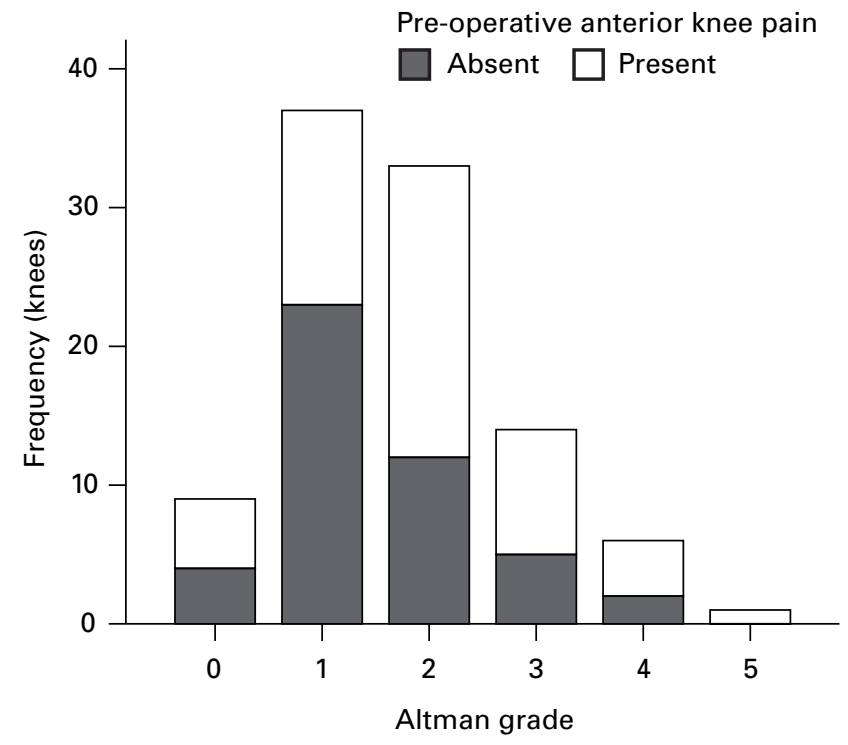

Fig. 3

Relationship between pre-existing anterior knee pain and the presence of any patellofemoral joint arthritis. Frequencies for each category (anterior knee pain present or absent) for each level of skyline radiological changes according to the Altman score is shown for 100 knees.

The fact that many patients had pre-existing anterior knee pain without evidence of degeneration of the patellofemoral joint and vice versa demonstrates the independence of these two conditions. Previous authors have shown only weak correlation between patellofemoral degenerative changes and anterior knee pain. ${ }^{12}$ Accordingly, it should not be assumed that the source of any anterior knee pain in patients undergoing UKR for medial compartment OA is degeneration of the patellofemoral joint. Localisation of pain is difficult, and anterior knee pain is a poorly-defined entity.

We found no evidence that degenerative changes seen pre-operatively on the skyline patellar views in the medial side of the patellofemoral joint compromised the outcome of the UKR; if anything they were associated with an improved outcome. This finding was echoed in our study of intra-operative assessment of the patellofemoral joint which is published in this issue. ${ }^{3}$ The reason for the improvement is uncertain, but the explanation for the changes in the medial side of the patellofemoral joint could be that they are a direct result of the medial tibiofemoral arthritis and thus corrected by the operation, or that the changes are a manifestation of ageing, are not associated with symptoms and are of no consequence.

The findings regarding degenerative changes of the lateral patellar facet are more difficult to interpret. We found that knees with lateral facet degeneration had a significantly worse outcome compared with knees without. However, the finding was not mirrored in all outcome measures, and the number of patients with severe lateral facet degeneration was small. A similar pattern and a similar inconsistency across variables was also reflected in our much larger study of intra-operative findings. ${ }^{3}$ It is clear that lateral facet degeneration requires extra consideration.

It is important to consider absolute as well as relative outcome. Although the data suggest that patients with lateral patellar degeneration have a worse outcome than those without, it also shows that patients with this pattern of degeneration did not have a poor outcome, with a mean two-year OKS of 37.6, and they did not have significant anterior knee pain. Therefore, lateral facet degeneration should perhaps be considered to be a relative, rather than an absolute, contraindication to UKR.

To make a definitive statement would require a randomised controlled trial to determine whether the outcome was better after TKR rather than UKR in these patients. As this subgroup of patients is small, such a study would be difficult to perform. Therefore, we recommend that caution be observed if there is radiological evidence of significant lateral patellar degeneration.

It is relevant that only a few patients with lateral patellofemoral joint space obliteration were included in this study and there were none with significant lateral patellar bone loss. If at operation significant bone loss with grooving was seen in the lateral patellofemoral joint, then a TKR was implanted rather than a UKR. Therefore, we have no outcome data for UKR implanted with significant lateral patellar bone loss. 
There is a concern that, even if anterior knee pain or degeneration of the patellofemoral joint on skyline radiographs does not affect the short-term clinical outcome, it might result in long-term failures. However, longer term reports in the literature of the mobile-bearing Oxford UKR, which have followed unchanged indications for surgery, have identified anterior knee pain or patellofemoral joint problems in a minimal number of cases even in those which have a relatively high instance of revision $(5 \%$ at 15 years). ${ }^{5,13-20}$

In conclusion, there is good evidence that neither preoperative anterior knee pain nor the presence of medial degeneration of the patellofemoral joint, as seen on skyline radiological views, should be a contraindication for medial Oxford UKR. The situation is less clear for lateral patellar changes, and a more cautious approach is advised. If there is lateral patellar bone loss and grooving we would implant a TKR.

The authors thank Dr H. S. Gill for his contribution to the database design, and Mrs B. Marks, research administrator.

The author or one or more of the authors have received or will receive benefits for personal or professional use from a commercial party related directly or indirectly to the subject of this article. In addition, benefits have been or will be directed to a research fund, foundation, educational institution, or other nonprofit organisation with which one or more of the authors is associated.

\section{References}

1. Kozinn S, Scott R. Surgical treatment of unicompartmental degenerative arthritis of the knee. Rheum Dis Clin North Am 1988;14:545-64.

2. Goodfellow J, $\mathbf{0}$ 'Connor J. Clinical results of the Oxford knee: surface arthroplasty of the tibiofemoral joint with a meniscal bearing prosthesis. Clin Orthop 1986;205:21-42.

3. Beard DJ, Pandit H, Gill HS, et al. The influence of the presence and severity of preexisting patellofemoral degenerative changes on the outcome of the Oxford medial unicompartmental knee replacement. J Bone Joint Surg [Br] 2007;89-B:1597-601.
4. Goodfellow J, O'Connor J, Dodd C, Murray D. Unicompartmental arthroplasty with the Oxford Knee. First ed. Oxford: Oxford University Press, 2006.

5. Price AJ, Webb J, Topf H, et al. Rapid recovery after Oxford unicompartmental arthroplasty through a short incision. J Arthroplasty 2001;16:970-6.

6. No authors listed. The Oxford unicompartmental knee system: surgical technique brochure. Warsaw: Biomet Orthopedics Inc., 2004:1-36.

7. Insall JN, Dorr LD, Scott RD, Scott WN. Rationale of the Knee Society clinical rating system. Clin Orthop 1989;248:13-14.

8. Dawson J, Fitzpatrick R, Murray D, Carr A. Questionnaire on the perceptions of patients about total knee replacement. J Bone Joint Surg [Br] 1998;80-B:63-9.

9. Ahlback S. Osteoarthritis of the knee: a radiographic investigation. Acta Radiol Diagn (Stockh) 1968;277(Suppl):7-72.

10. Altman RD, Fries JF, Bloch DA, et al. Radiographic assessment of progression in osteoarthritis. Arthritis Rheum 1987;30:1214-25.

11. Altman D. Practical statistics for medical research. London: Chapman and Hall/ CRC,1999.

12. Han I, Chang CB, Lee $\mathbf{S}$, et al. Correlation of the condition of the patellar articular cartilage and patellofemoral symptoms and function in osteoarthritic patients undergoing total knee arthroplasty. J Bone Joint Surg [Br]2005;87-B:1081-4.

13. Berger R, Meneghini R, Sheinkop $\mathbf{M}$, et al. The progression of patellofemoral arthrosis after medial unicompartmental replacement: results at 11 to 15 years. Clin Orthop 2004;428:92-9.

14. Emerson RH Jr, Higgins LL. A comparison of highly instrumented and minimally instrumented unicompartmental knee prostheses. Clin Orthop 2004;428:153-7.

15. Keys GW, UI-Abiddin Z, Toh EM. Analysis of first forty Oxford medial unicompartmental knee replacements from a small district hospital in the UK. Knee 2004;11:375-7.

16. Kumar A, Fiddian $\mathbf{N}$. Medial unicompartmental arthroplasty of the knee. Knee 1999;6:21-3.

17. Murray D, O'Connor J, Goodfellow J. The Oxford medial unicompartmental arthroplasty: a ten year survival study. J Bone Joint Surg [Br] 1998;80-B:983-9.

18. Pandit H, Jenkins C, Barker K, Dodd CA, Murray DW. The Oxford medial unicompartmental knee replacement using a minimally-invasive approach. J Bone Joint Surg [Br] 2006;88-B:54-60.

19. Rajasekhar C, Das S, Smith A. Unicompartmental knee arthroplasty: 2- to 12-year results in a community hospital. J Bone Joint Surg [Br] 2004;86-B:983-5.

20. Vorlat P, Putzeys G, Cottenie D, et al. The Oxford unicompartmental knee prosthesis: an independent 10-year survival analysis. Knee Surg Sports Traumatol Arthrosc 2006;14:40-5 